\title{
Suicidal Ideation in Underweight Adults Who Attempt to Lose Weight: Korea National Health and Nutrition Examination Survey, 2007-2012
} Original Article

\author{
Jinho Kim, Jinyoung Shin, Yun A Kim, Jungkwon Lee*
}

Department of Family Medicine, Samsung Medical Center, Sungkyunkwan University School of Medicine, Seoul, Korea

\section{ABSTRACT}

Background: Being underweight has been related to health risks. However, little is known about the relationship between suicidal ideation and attempting to lose weight. This study was conducted to examine if there is an association between suicidal ideation and attempting to lose weight among underweight adults.

Methods: A cross-sectional study of 1,122 underweight adults (range, 19 to 69 years) was conducted based on the Korea National Health and Nutrition Examination Survey, 2007-2012. We examined suicidal ideation, doctor-diagnosed depression, depressive mood, stress, physical activity, health-related behavior, comorbidity, and socioeconomic status by weight loss attempts. Logistic regression analysis was performed to examine the association between suicidal ideation and weight loss attempts. The following covariates were controlled for: age, sex, physical activity, alcohol problem, marital status, education, income, occupation, self-perception of body image, chronic disease, and body mass index.

Results: There were 101 subjects in the weight loss attempt group and 1,021 in the non-attempt group. The attempt group had a higher risk of suicidal ideation (odds ratio [OR], 2.47; 95\% confidence interval [CI], 1.35 to 4.53 ) and depressive symptoms (OR, 2.17; $95 \%$ CI, 1.19 to 3.98). After depressive symptoms were added to the covariates, the risk of suicidal ideation was also significant (OR 2.11, 95\% CI: 1.03 to 4.35). The two groups did not significantly differ in doctor-diagnosed depression and stress.

Conclusion: Weight loss attempts were associated with suicidal ideation in underweight Korean adults.

Keywords: Thinness; Weight Loss; Suicidal Ideation

Received: August 28, 2014, Accepted: February 25, 2015

*Corresponding Author: Jungkwon Lee

Tel: +82-2-3410-2441, Fax: +82-2-3410-0388, E-mail: Jungkwon.lee@samsung.com

Korean Journal of Family Medicine

Copyright (C) 2015 The Korean Academy of Family Medicine

This is an open-access article distributed under the terms of the Creative Commons Attribution Non-Commercial License (http://creativecommons.org/licenses/by-nc/3.0) which permits unrestricted noncommercial use, distribution, and reproduction in any medium, provided the original work is properly cited. 


\section{INTRODUCTION}

Being obese and being underweight have been related to higher mortality rates. ${ }^{1)}$ Obesity adversely affects health by increasing the risk of metabolic diseases, cancers, and inflammatory diseases. ${ }^{2)}$ The adverse health effects of being underweight include an increase in the susceptibility of infectious disease; moreover, underweight expectant mothers have higher perinatal risks; ${ }^{3)}$ however, the risks associated with being underweight are not as well-studied as the risks associated with obesity. Moreover, attention to the adverse effects of being underweight is scarce.

In the field of mental health, the association between body mass index (BMI) and suicide or depression is well-reported; actual BMI and self-perceptions of BMI have been attributed to both suicide and depression. ${ }^{4,5)}$ In addition, self-perceived attempts to control weight have also been related to them. ${ }^{\text {.) }}$

Obese or overweight patients are under a considerable amount of stress; they are also at greater risk for depression due to socio-cultural factors, ${ }^{7)}$ However, being underweight has been associated with suicide. ${ }^{8)}$ For instance, in a large cohort study, it was reported that the risk of depression increased as body weight increased; however, there was an inverse relation between suicide and body weight." ${ }^{9}$ Thus, being underweight may be a risk factor for suicide; indeed, it could raise the risk of suicide. Moreover, there could be a discordance between actual BMI, self-perception of BMI (body image misperception), and attempts to control weight.

According to the 2012 Korea National Health and Nutrition Examination Survey (KNHANES), the proportion of underweight people over 19 years of age was $5 \%$ (men $2.6 \%$, women $7.3 \%$ ). ${ }^{10)}$ To date, there have been studies that have focused on adolescents' weight control attempts and suicide, but these studies have not solely focused on underweight individuals. However, the pressure to be slim is not limited to a certain age and weight; indeed, underweight individuals may feel pressure to be skinny or to lose weight. ${ }^{11,12)}$

Therefore, the aim of this study was to determine the factors that affect suicide in underweight adults. It was hypothesized that actual weight loss attempts rather than perceptions of body image would be directly related to suicide. Thus, we examined the association between weight loss attempts and suicidal ideation in underweight adults.

\section{METHODS}

\section{Population}

A cross-sectional study was conducted based on data from the KNHANES, 2007-2012; the KNHANES was conducted by the Korea Centers for Disease Control and Prevention. Specifically, a sample survey targeting the entire non-institutionalized civilian population was conducted with a complex, stratified, multistage, cluster-sampling design. ${ }^{10)}$ Subjects included in the KNHANES, 2007-2012 were 50,405 individuals aged $\geq 1$ year. Of these individuals, 32,222 were 19 to 69 years. 
Subjects with no anthropometric data $(\mathrm{n}=1,980)$ and pregnant women $(\mathrm{n}=214)$ were excluded; this resulted in 30,028 subjects remaining (12,925 men and 17,103 women). Among the remaining subjects, 1,338 were underweight; however, 216 were excluded because they failed to complete all of the questionnaires. Therefore, 1,122 (148 men and 974 women) were included in the analysis. This study was approved by institutional review board of Samsung Medical Center (IRB file no.: 2014-07-012).

\section{Measurement}

All anthropometric measurements and surveys were conducted in mobile examination centers by trained health professionals. The self-administered surveys included questions about health behaviors such as smoking, drinking, physical activity, and weight control. Data on socioeconomic status and history of chronic disease or cancer were obtained through face-to-face interviews.

Anthropometric measurements were taken while participants wore light clothing and no shoes. Height was measured to the nearest $0.1 \mathrm{~cm}$ (range, 6 to $230 \mathrm{~cm}$ ) using a SECA-225 apparatus (SECA Deutschland, Hamburg, Germany). Weight was measured to the nearest $0.1 \mathrm{~kg}(\leq 200 \mathrm{~kg}$ ) using a GL6000-20 apparatus (CASKOREA, Seoul, Korea). BMI was calculated by dividing weight ( $\mathrm{kg}$ ) by the square of height $\left(\mathrm{m}^{2}\right)$; an individual was considered underweight if there BMI was $<18.5 \mathrm{~kg} / \mathrm{m}^{2}$.

The study population was divided into a weight loss attempt group and a non-attempt group; this was based on the questionnaires (e.g., weight loss attempt vs. maintain, gain, and no attempt during the past one year). Body self-perception was answered via five items (very thin, somewhat thin, average, somewhat obese, and very obese); these were classified into thin, average, or obese. Physical activity was calculated by the Korean version of the International Physical Activity Questionnaire (IPAQ) short form; ${ }^{13)}$ it was calculated as a continuous score (unit: metabolic equivalents [MET]-min/wk) and was classified into three categories. High physical activity was any one of the following 2 criteria (vigorousintensity activity on at least 3 days and accumulating at least 1,500 MET-min/wk or 7 or more days of any combination of walking, moderate-intensity or vigorous intensity activities achieving a minimum of at least 3,000 MET-min/wk). Moderate physical activity was any one of the following 3 criteria (3 or more days of vigorous activity of at least 20 minutes per day, 5 or more days of moderate-intensity activity and/or walking of at least 30 minutes per day, 5 or more days of any combination of walking, moderate-intensity or vigorous-intensity activities achieving a minimum of at least 600 MET-min/ wk. Low physical activity was the lowest level not to meet above two criteria (IPAQ: http://www.ipaq. ki.se). Smoking was classified into current and noncurrent smokers. A calculated AUDIT (alcohol use disorders identification test) score was used to define alcohol use disorder; this was defined as a score of more than 16 points. ${ }^{14)}$ Marital status was classified into married, unmarried and other (divorced, separated, and widowed). Education level was classified into high (more than high school graduate) and low (less than middle school). Income was categorized by quartiles; subjects were classified as high or low. Occupation was classified by the Korean Standard Classification of Occupations revised in 2007; specifically, individuals were categorized as non-manual workers, manual workers, and other (students, 
housewives, and unemployed; soldiers were excluded). Chronic disease was diagnosed by a doctor and was defined as hypertension, dyslipidemia, diabetes, stroke, ischemic heart disease, arthritis, asthma, liver cirrhosis, and renal failure. Doctor-diagnosed cancers of the stomach, liver, colon, breast, cervix, lung, thyroid, and other organs were also reported. Individuals who had been previously diagnosed by a doctor as having any kind of cancer were considered as having a history of cancer.

Indicators related to mental health were defined as doctor-diagnosed depression, depressive symptoms lasting more than two consecutive weeks, suicidal ideation during the past year, any suicidal attempts during the past year, and the degree of stress awareness.

\section{Statistical Analysis}

Complex samples analysis was used to analyze the KNHANES raw data; IBM SPSS ver. 21.0 (IBM Co., Armonk, NY, USA) was used. Data were expressed as mean \pm standard error or percentage \pm standard error. The unweighted number of each variable was not shown. Chi-square tests and Student t-tests were used to analyze every component by weight loss attempt. Logistic regression analysis was used to control for the significant factors known to influence suicidal ideation or depression. There was no cancer diagnosis in the weight loss attempt group; thus, it was subsequently excluded from the logistic regression model. Depressive symptoms are known to be a weighty factor in similar studies; therefore, depressive symptoms were added to model 2 for sensitivity analysis. Differences were considered to be statistically significant when the $\mathrm{P}$-value was $<0.05$. The prevalence of suicidal ideation in adults was about $15 \%{ }^{10)}$ and the assumption of the control group was about $30 \%$. Therefore, if alpha was 0.05 , beta was 0.8 , and the proportion between two groups was 0.1 , adequate sample sizes were 667 for the non-attempt group and 67 for the attempt group.

\section{RESULTS}

Among the 1,122 subjects analyzed, the weight loss attempt group comprised 101 subjects and the non-attempt group comprised 1,021 subjects. The general characteristics of the underweight adults by weight loss attempt group are shown in Table 1 . The mean age of the attempt group was significantly younger than the non-attempt group $(27.5 \pm 0.8,32.8 \pm 0.9$, respectively). The mean BMI of the attempt group and the non-attempt group was $17.9 \pm 0.1$ and $17.6 \pm 0.1$, respectively; the attempt group had significantly higher BMI than the non-attempt group. In addition, there were more women in the attempt group than the non-attempt group $(97.4 \%$ vs. $83.0 \%, \mathrm{P}=0.005)$. For self-perceptions, the attempt group had a higher likelihood of perceiving themselves as not thin $(63.2 \%$ vs. $19.1 \%$; odds ratio [OR], 7.279; 95\% confidence interval [CI], 4.451 to 11.905$)$. The attempt group had a higher level of physical activity and education than the non-attempt group. There was no significant difference between the two groups in smoking status, alcohol problem, and income. Unadjusted mental health indicators are presented in Table 2. The attempt group had a higher likelihood of suicidal ideation and depressive symptoms as well as a higher level of stress than the non-attempt group (OR, 2.86; 95\% CI, 
Jinho Kim et al: Weight Loss Attempts in Underweight and Suicidal Ideation

1.76 to 4.65 ; OR, 2.40 ; $95 \%$ CI, 1.45 to 3.96 ; OR, 2.01 ; $95 \%$ CI, 1.26 to 3.22 , respectively).

Table 1. General characteristics of underweight adults by weight loss attempt group

\begin{tabular}{|c|c|c|c|}
\hline Characteristic & No attempt (unweighted $\mathrm{n}=1,021$ ) & Attempt (unweighted $\mathrm{n}=101$ ) & P-value ${ }^{*}$ \\
\hline Age (y) & $32.8 \pm 0.9$ & $27.5 \pm 0.8$ & 0.001 \\
\hline Age group (y) & & & $<0.001$ \\
\hline $19-39$ & $76.8(1.6)$ & $93.8(2.8)$ & \\
\hline $40-69$ & $23.2(1.6)$ & $6.4(2.8)$ & \\
\hline Sex & & & 0.005 \\
\hline Women & $83.0(1.5)$ & $97.4(2.1)$ & \\
\hline Men & $17.0(1.5)$ & $2.6(2.1)$ & \\
\hline Body mass index $\left(\mathrm{kg} / \mathrm{m}^{2}\right)$ & $17.6 \pm 0.1$ & $17.9 \pm 0.1$ & $<0.001$ \\
\hline Self-perception & & & $<0.001$ \\
\hline Thin & $80.9(1.6)$ & $36.8(5.4)$ & \\
\hline Average or obese & $19.1(1.6)$ & $63.2(5.4)$ & \\
\hline MET-min/wk ${ }^{\dagger}$ & $5,625 \pm 981$ & $5,262 \pm 950$ & 0.73 \\
\hline Physical activity category ${ }^{\dagger}$ & & & 0.02 \\
\hline Low & $68(1.8)$ & $52.4(5.8)$ & \\
\hline Moderate & $21.4(1.5)$ & $30.3(5.0)$ & \\
\hline High & $10.6(1.1)$ & $17.3(4.5)$ & \\
\hline Current smoking & & & 0.41 \\
\hline No & $81.1(1.6)$ & $84.9(4.0)$ & \\
\hline Yes & $18.9(1.6)$ & $15.1(4.0)$ & \\
\hline Alcohol problem & & & 0.71 \\
\hline No & $93.6(1.1)$ & $92.4(3.2)$ & \\
\hline Yes & $6.4(1.1)$ & $7.6(3.2)$ & \\
\hline Marital status & & & 0.005 \\
\hline Married & $48.7(2.1)$ & $30.0(5.0)$ & \\
\hline Unmarried & $47.5(2.1)$ & $67.4(5.2)$ & \\
\hline Other $^{\S}$ & $3.8(0.7)$ & $2.6(1.9)$ & \\
\hline Education & & & 0.001 \\
\hline$\leq$ Middle school & $10.3(1.1)$ & $1.8(1.0)$ & \\
\hline$\geq$ High school & $89.7(1.1)$ & $98.2(1.0)$ & \\
\hline Income & & & 0.26 \\
\hline Low & $36.8(2.1)$ & $30.3(5.2)$ & \\
\hline High & $63.8(.2 .1)$ & $69.7(5.2)$ & \\
\hline Occupation & & & 0.14 \\
\hline Non-manual & $42.5(1.9)$ & $56.5(5.1)$ & \\
\hline Manual & $12.9(1.3)$ & $3.2(2.2)$ & \\
\hline Other" & $44.6(1.9)$ & $40.2(5.0)$ & \\
\hline Chronic disease & & & 0.031 \\
\hline No & $93.8(0.8)$ & $99.0(1.0)$ & \\
\hline Yes & $6.2(0.8)$ & $1.0(1.0)$ & \\
\hline Cancer & & & 0.252 \\
\hline No & $98.5(0.4)$ & $100.0(0)$ & \\
\hline Yes & $1.5(0.4)$ & 0 & \\
\hline
\end{tabular}

Values are presented as mean \pm standard error or $\%$ (SE).

${ }^{*}$ By t-test or chi-square test. ${ }^{\dagger}$ Metabolic equivalents assessed by International Physical Activity Questionnaire. ${ }^{\star} \mathrm{Al}-$ cohol problem means AUDIT (alcohol use disorders identification test) score $\geq 16$. \$ncluding divorced, separated, and widowed. \| Including students, housewives, and unemployed persons (soldiers were excluded). 
Table 2. Mental health of underweight adults (unadjusted)

\begin{tabular}{|c|c|c|c|c|}
\hline Characteristic & $\begin{array}{c}\text { No attempt } \\
\text { (unweighted } n=1,021 \text { ) }\end{array}$ & $\begin{array}{c}\text { Attempt } \\
\text { (unweighted } n=101 \text { ) }\end{array}$ & $\begin{array}{c}\text { Odds ratio } \\
\text { (95\% confidence interval) }\end{array}$ & P-value* \\
\hline Suicidal ideation & & & $2.86(1.76-4.65)$ & $<0.001$ \\
\hline No & $83.8(1.4)$ & $64.5(5.4)$ & & \\
\hline Yes & $16.2(1.4)$ & $35.5(5.4)$ & & \\
\hline Suicidal trial & & & No available & 0.209 \\
\hline No & $94.0(2.2)$ & $100(0)$ & & \\
\hline Yes & $6.0(2.2)$ & 0 & & \\
\hline Doctor-diagnosed depression & & & $0.28(0.06-1.33)$ & 0.087 \\
\hline No & $90.5(1.0)$ & $97.1(2.2)$ & & \\
\hline Yes & $9.5(1.0)$ & $2.9(2.2)$ & & \\
\hline Depressive symptoms & & & $2.40(1.45-3.96)$ & 0.001 \\
\hline No & $86.2(1.3)$ & $72.3(4.7)$ & & \\
\hline Yes & $13.8(1.3)$ & $27.7(4.7)$ & & \\
\hline Stress & & & $2.01(1.26-3.22)$ & 0.003 \\
\hline Low & $68.2(1.8)$ & $51.6(5.4)$ & & \\
\hline High & $31.8(1.8)$ & $48.4(5.4)$ & & \\
\hline
\end{tabular}

Values are presented as \% (standard error).

*By chi-square test.

The groups did not significantly differ on doctor-diagnosed depression. Suicidal trial was '0' in the attempt group; therefore, it was not available for analysis. The factors that were independently related to weight loss attempts and were not related in this study were included in the logistic regression analysis (model 1) (Table 3); however, other studies have indicated that these factors are relevant. The significance of suicidal ideation and depressive symptoms were maintained (OR, 2.47; 95\% CI, 1.35 to 4.53 ; OR, 2.17 ; $95 \%$ CI, 1.19 to 3.98 , respectively). Doctor-diagnosed depression remained non-significant. The odds ratio of suicidal ideation after adjusting for depressive symptoms (model 2) decreased slightly, but remained statistically significant (OR, 2.11; 95\% CI, 1.03 to 4.35).

Table 3. Results of multiple logistic regression analyses on weight loss attempts

\begin{tabular}{|c|c|c|c|c|c|c|c|c|}
\hline \multirow{2}{*}{ Adults } & \multicolumn{2}{|c|}{ Suicidal ideation } & \multicolumn{2}{|c|}{ Doctor-diagnosed depression } & \multicolumn{2}{|c|}{ Depressive symptom } & \multicolumn{2}{|c|}{ Stress } \\
\hline & Non-attempt ${ }^{*}$ & ${ }^{*}$ Attempt ${ }^{*}$ & Non-attempt & Attempt & Non-attempt & Attempt & Non-attempt & Attempt \\
\hline Model $1^{\dagger}$ & 1.00 & $\begin{array}{c}2.47 \\
(1.35-4.53)\end{array}$ & 1.00 & $\begin{array}{c}0.36 \\
(0.65-1.73)\end{array}$ & 1.00 & $\begin{array}{c}2.17 \\
(1.19-3.98)\end{array}$ & 1.00 & $\begin{array}{c}1.58 \\
(0.93-2.69)\end{array}$ \\
\hline Model 2 & 1.00 & $\begin{array}{c}2.11 \\
(1.03-4.35)\end{array}$ & ND & ND & ND & ND & ND & ND \\
\hline
\end{tabular}

Values are presented as odds ratio ( $95 \%$ confidence interval).

ND: not done.

${ }^{*}$ Unweighted no. of attempt $=1,021$; non-attempt $=101$. ${ }^{\dagger}$ Model 1: adjusted by age group, sex, physical activity category, alcohol problem, marital status, education, income, occupation, self-perception, chronic disease, and body mass index. Model 2: adjusted by model 1 and depressive symptom. 


\section{DISCUSSION}

This study was conducted to examine the effect of attempting to lose weight on suicidal ideation. We found that attempting to lose weight was significantly associated with suicidal ideation in underweight adults; this finding remained significant after adjusting for depressive symptoms. In previous studies, the misperception of body image has been related to suicidal ideation; these studies have indicated both an underestimation and overestimation. ${ }^{5)}$ Attempts to control weight appear to be influenced by the misperception of body image. ${ }^{15)}$ The findings of the current study indicated that attempting to lose weight was associated with suicidal ideation when controlling for body image misperception. Therefore, these findings suggest that attempting to lose weight is not just a consequence of misperceiving body image but also has an independent influence on suicidal ideation.

Other studies, including one using the 10-12 KNHANES, have shown that weight loss attempts in men and weight maintenance attempts in women were associated with suicidal ideation, after controlling for actual body weight and body image misperception; ${ }^{6}$ this finding is consistent with the findings reported herein. However, there was no association between BMI and suicidal ideation. There are several, large cohort studies that have showed an inverse relationship between BMI and suicide. For instance, in Norway, 74,332 people were followed for 20 years; results indicated that lower BMI was associated with a higher rate of suicide. ${ }^{9)}$ In addition, 1,133,019 Swedish individuals born between 1956 and 1976 were observed for 14 years; this study indicated that suicide attempts increased with decreasing BMI. After controlling for depression in the underweight group, the risk of suicide attempts still remained significant in underweight individuals. ${ }^{16)}$ Moreover, in a study of 4 million United States veterans, a relation between suicidal death and being underweight was reported. ${ }^{17)}$ Furthermore, in a study following 800,000 people for up to seven years in the UK, results indicated that being underweight was related to suicide attempts. However, this finding was no longer significant after controlling for depression. ${ }^{18)}$

In sum, there have been inconsistent results regarding the association between being underweight and suicide. However, it has generally been found that being underweight is a risk factor for suicide, regardless of other socio-cultural factors or mental health problems (e.g., depression). This may be attributed to the fact that lower cholesterol levels affect the metabolism of serotonin and impulsiveness. ${ }^{19)}$ For instance, in animal experiments, depression and stress were affected by leptin secreted in adipocytes. ${ }^{20)}$ Furthermore, a case-control study of humans confirmed that individuals who attempted suicide had lower cholesterol levels. ${ }^{21)}$

Depressive symptoms were significantly different in the weight loss attempt group and the nonattempt group; however, the two groups did not differ significantly in the diagnosis of depression. This may be the result of a discrepancy between the ratio of prevalence and actual diagnosis. ${ }^{22)}$ In addition, it was recently reported that the prevalence of depression was $6.7 \%{ }^{23)}$ however, it was $2.9 \%$ in the current study. It is possible that there was a selection bias in this population; there also may be no difference in the diagnosis of depression. The non-attempt group had higher physical activity; thus, it may be that physical activity influenced depressive symptoms in a way that prevented depression. ${ }^{24)}$ Therefore, the 
lack of difference in stress can be explained; however, that possibility seemed low. Therefore, a followup study seems necessary to examine differences in depression diagnosis.

A cross-sectional study in Korea analyzed the characteristics of individuals who lose weight when they are already underweight. These results indicated that underweight women had more attempts to lose weight; this was associated with a misperception of body image, a higher income, and being unmarried. ${ }^{11)}$ There were similar socioeconomic characteristics between the two groups in the current study; this is likely due to socio-cultural pressure to be thin as depicted in the media. This may result in underweight individuals losing weight. ${ }^{25,26)}$

Previous studies have separated men and women. However, in this study, the subjects were predominantly women; therefore, outcome variables did not change after conducting subgroup analysis by sex. Thus, the results of the entire population were reported.

The BMI of the attempt group was higher than the non-attempt group $(17.9,17.6)$; ideal BMI reported by high school girls was $17.3 .^{27)}$ This is under 18.5; thus, the difference in the decision to lose weight may depend on the degree of BMI, even among underweight individuals.

The current study used data targeting an entire population; this data was highly representative of Korea and there were ample subjects to include in the analysis. This is the first study to focus only on underweight adults. Importantly, attempts to lose weight were significantly associated with suicidal ideation after controlling for depressive symptom.

The current study had some limitations that require discussion. The association reported herein was from a cross-sectional study. Therefore, it was not possible to establish the exact pathophysiology linking weight loss attempts among underweight and suicidal ideation. The difference between subjects' current condition and the survey results may also exist since subjects reported about themselves within the past year. There are also a variety of clinical forms of suicide; these include ideation, attempt, or actual death. The current study only analyzed suicidal ideation because the number of suicide attempts was not large enough to include in the analysis. However, the findings reported herein do have clinical implications given that $30 \%$ of individuals that report suicidal ideation either plan their own suicide or attempt suicide within one year. ${ }^{28)}$ Finally, other mental illnesses associated with being underweight and suicide (e.g., anorexia nervosa) were not considered herein; however, we believe that these would have a minimal effect because the response rate for mental health counseling was small.

In this study, we found that weight loss attempts among underweight adults were associated with suicidal ideation; this finding was independent of other factors such as depressive symptoms, socioeconomic factors, and health factors. This study may be helpful in assessing suicidal risk; further studies are needed to determine the causal relations between the variables examined herein.

\section{CONFLICT OF INTEREST}

No potential conflict of interest relevant to this article was reported. 


\section{ACKNOWLEDGMENTS}

We would like to thank the Korea Centers for Disease Control and Prevention for the data obtained from the Korean National Health and Nutrition Examination Survey.

\section{REFERNSCES}

1. Flegal KM, Graubard BI, Williamson DF, Gail MH. Excess deaths associated with underweight, overweight, and obesity. JAMA 2005;293:1861-7.

2. Aronne LJ. Classification of obesity and assessment of obesity-related health risks. Obes Res 2002;10 Suppl 2:105S-15S.

3. World Health Organization. The world health report 2002: reducing risks, promoting healthy life. Geneva: World Health Organization; 2002.

4. Kim DS, Cho Y, Cho SI, Lim IS. Body weight perception, unhealthy weight control behaviors, and suicidal ideation among Korean adolescents. J Sch Health 2009;79:585-92.

5. Eaton DK, Lowry R, Brener ND, Galuska DA, Crosby AE. Associations of body mass index and perceived weight with suicide ideation and suicide attempts among US high school students. Arch Pediatr Adolesc Med 2005;159:513-9.

6. Jeon H, Lee K, Kim HJ, Lee YM, Kim JW. The association between weight-related attitudes and suicidal ideation in adults. J Korean Neuropsychiatr Assoc 2014;53:114-21.

7. Luppino FS, de Wit LM, Bouvy PF, Stijnen T, Cuijpers P, Penninx BW, et al. Overweight, obesity, and depression: a systematic review and meta-analysis of longitudinal studies. Arch Gen Psychiatry 2010;67:220-9.

8. Mukamal KJ, Rimm EB, Kawachi I, O'Reilly EJ, Calle EE, Miller M. Body mass index and risk of suicide among one million US adults. Epidemiology 2010;21:82-6.

9. Bjerkeset O, Romundstad P, Evans J, Gunnell D. Association of adult body mass index and height with anxiety, depression, and suicide in the general population: the HUNT study. Am J Epidemiol 2008;167:193-202.

10. Ministry of Health and Welfare. The fifth Korea National Health and Nutrition Examination Survey (KNHANES V-2, V-3) [Internet]. Cheongju: Korea Centers for Disease Control and Prevention [cited 2014 Jan 3]. Available from: http://knhanes.cdc.go.kr/knhanes/index.do.

11. Choi OJ, Cho YG, Kang JH, Park HA, Kim KW, Hur YI, et al. Weight control attempts in underweight Korean adults: Korea National Health and Nutrition Examination Survey, 2007-2010. Korean J Fam Med 2013;34:393-402.

12. McElhone S, Kearney JM, Giachetti I, Zunft HJ, Martinez JA. Body image perception in relation to recent weight changes and strategies for weight loss in a nationally representative sample in the European Union. Public Health Nutr 1999;2(1A):143-51.

13. Oh JY, Yang YJ, Kim BS, Kang JH. Validity and reliability of Korean version of International Physical 
Activity Questionnaire (IPAQ) short form. J Korean Acad Fam Med 2007;28:532-41.

14. Babor TF, Higgins-Biddle JC, Saunders JB, Monteiro MG. The alcohol use disorders identification test: guidelines for use in primary care. 2nd ed. Geneva: World Health Organization; 2001.

15. Kim JS, Lee K. The relationship of weight-related attitudes with suicidal behaviors in Korean adolescents. Obesity (Silver Spring) 2010;18:2145-51.

16. Batty GD, Whitley E, Kivimaki M, Tynelius P, Rasmussen F. Body mass index and attempted suicide: Cohort study of 1,133,019 Swedish men. Am J Epidemiol 2010;172:890-9.

17. McCarthy JF, Ilgen MA, Austin K, Blow FC, Katz IR. Associations between body mass index and suicide in the veterans affairs health system. Obesity (Silver Spring) 2014;22:269-76.

18. Gao S, Juhaeri J, Reshef S, Dai WS. Association between body mass index and suicide, and suicide attempt among British adults: the health improvement network database. Obesity (Silver Spring) 2013;21:E334-42.

19. Evans J, Reeves B, Platt H, Leibenau A, Goldman D, Jefferson K, et al. Impulsiveness, serotonin genes and repetition of deliberate self-harm (DSH). Psychol Med 2000;30:1327-34.

20. Lu XY, Kim CS, Frazer A, Zhang W. Leptin: a potential novel antidepressant. Proc Natl Acad Sci U S A 2006;103:1593-8.

21. Diaz-Sastre C, Baca-Garcia E, Perez-Rodriguez MM, Garcia-Resa E, Ceverino A, Saiz-Ruiz J, et al. Low plasma cholesterol levels in suicidal males: a gender- and body mass index-matched case-control study of suicide attempters and nonattempters. Prog Neuropsychopharmacol Biol Psychiatry 2007;31:901-5.

22. Mitchell AJ, Vaze A, Rao S. Clinical diagnosis of depression in primary care: a meta-analysis. Lancet 2009;374:609-19.

23. Jeon HJ. Epidemiologic studies on depression and suicide. J Korean Med Assoc 2012;55:322-8.

24. Strohle A. Physical activity, exercise, depression and anxiety disorders. J Neural Transm 2009;116:777-84.

25. Owen R, Spencer RM. Body ideals in women after viewing images of typical and healthy weight models. Body Image 2013;10:489-94.

26. Andrade FC, Raffaelli M, Teran-Garcia M, Jerman JA, Garcia CA; Up Amigos 2009 Study Group. Weight status misperception among Mexican young adults. Body Image 2012;9:184-8.

27. Lee HK. Factors influencing need for cosmetic surgery among high school girls. Korean J Women Health Nurs 2011;17:126-36.

28. Nock MK, Borges G, Bromet EJ, Alonso J, Angermeyer M, Beautrais A, et al. Cross-national prevalence and risk factors for suicidal ideation, plans and attempts. Br J Psychiatry 2008;192:98-105. 